\title{
Long-term follow-up of planned treatment of spontaneous pneumothorax
}

\author{
IVAN LICHTER
}

\author{
Department of Surgery, University of Otago Medical School, Dunedin, New Zealand
}

\begin{abstract}
Lichter, I. (1974). Thorax, 29, 32-37. Long-term follow-up of planned treatment of spontaneous pneumothorax. Ninety-six patients presenting with spontaneous pneumothorax have been followed for a period of five to 12 years after initial treatment. Treatment was designed to deal with the pneumothorax episode and management was planned to minimize the risk of recurrence. Patients were treated initially by intercostal tube drainage, and subsequent management depended upon the nature of the underlying disease and the behaviour of the pneumothorax.

Two groups of patients were identified. Group A were young fit subjects with disease confined to the apex of the lung. Group B were older subjects with overt, often widespread pulmonary disease.

In the first group of young fit patients, early wedge resection was advised for patients at greatest risk of recurrence-those who had suffered a previous pneumothorax and those in whom the leak had persisted for more than $\mathbf{4 8}$ hours. Limited wedge resection removes the whole of the diseased tissue, leaves normal lung uncompromised, and is uniformly successful.

In the group of older patients, extensive disease precludes the use of the same safe and effective treatment. For this reason tube drainage was frequently continued for up to 14 days. Failure of treatment by tube drainage was treated by excision of bullae in those patients who were fit for thoracotomy, and by pleurodesis in the remainder.

Intercostal tube drainage alone was successful in $77 \%$ of group A patients and in $52.5 \%$ of group B patients so treated. With planned management as recommended, $95 \%$ of group A and $87 \%$ of group B patients were either cured by tube drainage alone or treated definitively and lastingly during their first admission to hospital.
\end{abstract}

Spontaneous pneumothorax is treated by a variety of methods. Some clinicians manage spontaneous pneumothorax by observation alone and await re-expansion of the lung (Hyde, 1962); others treat by thoracentesis (Carr, Silver, and Ellis, 1963). Tube thoracostomy is favoured by some (Wolcott, Shaver, and Jennings, 1963), chemical pleurodesis (Sengupta, 1963) and pleurectomy (Saha, 1964) by others. Thoracotomy and surgical excision of bullae is practised (MacQuigg, 1955) and even bilateral thoracotomy has been recommended on the basis of the expected occurrence of lesions in the contralateral lung (Baronofsky et al., 1957).

The liability to recurrence of pneumothorax is an important consideration in management and it is desirable that there should be a plan of treatment that will provide for the individual patient

Requests for reprints: Ivan Lichter, F.R.C.S., Department of Surgery, University of Otago Medical School, Dunedin, New a system of management which takes into account the likelihood of recurrence. The recurrence rate is affected by a number of factors. It is contingent upon the nature of treatment used; Killen and 9 Gobbel (1968) tabulate results from a number of sources and show that in all types of pneumothorax, tube thoracostomy is followed by a lower $N$ recurrence rate $(16 \%)$ than observation or thoracentesis alone $(26 \%)$. There is evidence that in $N$ patients with primary spontaneous pneumothorax a higher recurrence rate can be expected in those who have suffered one or more previous episodes $c$ than in patients experiencing their first pneumo- $\frac{D}{\mathbb{D}}$ thorax (Gobbel, Rhea, Nelson, and Daniel, 1963). $\stackrel{\oplus}{+}$ Our own experience has also indicated that when $\square$ such a pneumothorax fails to seal within $\mathbf{4 8}$ hours, the likelihood of recurrence is high. These factors identify the patients in whom pneumothorax is likely to recur. Treatment can then be planned so that, where indicated, a decision on definitive 
therapy can be taken early, so avoiding unnecessary readmission to hospital for recurrences.

Two distinct groups of patients suffering from spontaneous pneumothorax can be identified.

Group A comprises young and fit patients, usually male, who have been in good health prior to the first episode of spontaneous pneumothorax. Chest radiographs frequently show no abnormality, although occasionally apical bullous cysts may be distinguished. This type of spontaneous pneumothorax has been variously referred to in the literature as primary spontaneous pneumothorax, idiopathic pneumothorax, benign pneumothorax, and a number of other descriptions (Killen and Gobbel, 1968).

Lichter and Gwynne (1971) showed that in this group of patients the disease process is limited to a small area at the apex of the lung, and the remainder of the lung is completely normal. The apical lesion consists of an area of fibrosis surmounted by bullous cysts of varying size, and it is from a perforation or rupture of one of these cysts that the air leak arises.

Group B patients often exhibit widespread and serious lung disease that is clinically and radiologically apparent. This group has been designated secondary spontaneous pneumothorax. The air leak arises from bullous cysts associated with the underlying disease process. This group largely comprises patients with chronic bronchitis and emphysema but also includes patients with old healed tuberculosis where fibrosis has resulted in bullous formation. A variety of other conditions, such as bronchiectasis, lung tumours, staphylococcal pneumonia, lung infarction, silicosis, congenital cysts and endometriosis, may also occasionally be complicated by spontaneous pneumothorax.

For reasons of safety, all patients were treated initially by intercostal tube drainage. Thereafter the differing aetiology suggested the need for separate management plans for spontaneous pneumothorax occurring in young fit patients with very localized lung disease, and for the group of patients, generally of an older age group, with manifest, more widespread or serious lung disease. In group A patients definitive surgery was advised when the recurrence risk was deemed to be high. The localized nature of the disease process in this group of patients allows of a relatively limited operative procedure, which removes all the diseased area, so precluding the possibility of recurrence, and moreover avoids interference with normal lung.

In older patients with extensive lung disease (group B) a limited curative resection is not feasible. Moreover these patients are often respiratory cripples in whom thoracotomy may not be feasible.

A series of patients has been treated on these principles; the protocol is outlined below.

\section{PATIENTS STUDIED AND METHODS}

Ninety-six patients with spontaneous pneumothorax admitted to the Thoracic Surgical Unit during the period of 1961-68 and treated according to the protocol described below have been followed for from five to 12 years. On admission all patients were treated by tube thoracostomy with the exception of three who had a minimal pneumothorax.

In the group of young fit subjects (group A) the air leak had usually ceased within 24 hours. At this time, or 24 hours after cessation of air leak, a chest film was taken to ensure that lung expansion was complete, after which the intercostal drainage tube was removed. Twenty-four hours later a check film was taken to ensure that lung expansion had been maintained and the patient was discharged from hospital. Tube management was abandoned if the leak persisted for more than 48 hours despite adequate tube drainage, and arrangements were made for early operation. Early surgery was also undertaken if there was a history of previous episodes of spontaneous pneumothorax.

At operation the small diseased area at the apex of the lung was removed by wedge resection (Lichter and Gwynne, 1971). The remainder of the lung was invariably healthy, and pleurodesis or pleurectomy was not employed.

In the group of older patients with extensive underlying lung disease (group B), a trial period of tube drainage was usually employed and this was continued for up to 14 days. If this failed, thoracotomy with excision of bullae was undertaken in those considered fit for such a procedure; the remainder were treated by chemical pleurodesis using kaolin powder inserted via an intercostal cannula. An early decision regarding excision of bullae was taken when there was a history of recurrence or when a gross air leak or failing respiratory function indicated the need for rapid lung re-expansion.

At operation all surface pleural blebs, bullae or cysts were excised, bronchial fistulae were closed, and leaks were oversewn. Because it was not possible to ensure that all diseased areas were adequately dealt with, pleurodesis was sometimes used. Pleural abrasion with a dry swab produced an effective result without the pain and excessive pleural effusion associated with pleurodesis by chemical irritants.

Where bilateral spontaneous pneumothorax occurred, each side was treated on its own merits according to the above plan. 
RESULTS

Of 154 patients treated in the Thoracic Surgical Unit between 1961 and 1968, 96 patients were managed according to the foregoing protocol and were available for follow-up five to 12 years after entry into the series. A number of elderly patients had died of chronic obstructive respiratory disease, some from other illnesses, and a few were lost to follow-up. The distribution of patients by group is shown in Table $\mathrm{I}$.

\section{T A B L E I}

\begin{tabular}{c|c|c}
\hline Group & No. of Patients & \\
\hline A & $42(1$ bilateral) & 43 sides \\
B & $54(6$ bilateral) & 60 sides \\
\hline Total & 96 & 103 sides \\
\hline
\end{tabular}

GROUP A There were 38 male and four female patients in the series.

Age range Thirty-four patients were aged 1630 years. Eight patients were aged $30-47$ years. Out of five females in the group, three were in their fourth decade.

Side affected The right side was affected in 21 , and the left in 22 episodes.

Management (Tables II to IV) Intercostal tube drainage was followed by wedge resection when air leak continued for more than 48 hours. Wedge resection was undertaken as a primary measure in patients who were admitted with a recurrent pneumothorax.

Late death There was one death in the series. The patient died of lung cancer six years after treatment of the spontaneous pneumothorax by intercostal tube.

T A B LE I I

GROUP A: INITIAL TREATMENT OF 43 PNEUMOTHORACES

\begin{tabular}{|c|c|c|}
\hline Treatment & No. & Recurrence \\
\hline $\begin{array}{l}\text { None (observation only) } \ldots \text {. } \\
\text { Intercostal tube drainage only } \\
\text { Intercostal tube drainage followed by wedge } \\
\text { resection for persisting leak } \\
\text { Wedge resection for history of recurrence }\end{array}$ & $\begin{array}{r}2 \\
22 \\
4 \\
15\end{array}$ & $\begin{array}{l}0 \\
2^{1} \\
0 \\
0\end{array}$ \\
\hline Total & 43 & 2 \\
\hline
\end{tabular}

1Both recurrences after intercostal tube drainage occurred within five months of initial treatment.
T A B L E I I I

GROUP A: OUTCOME OF 26 PNEUMOTHORACES TREATED BY INTERCOSTAL TUBE DRAINAGE

\begin{tabular}{lll|r}
$\begin{array}{l}\text { Succeeded as only treatment } \\
\text { Continued air leak requiring wedge resection }\end{array}$ & $\ldots$ \\
$\begin{array}{l}\text { Late recurrence of pneumothorax (subsequently } \\
\text { treated by wedge resection) }\end{array}$ & $\ldots$ & $\ldots$ & $\ldots$
\end{tabular} \mid $\begin{array}{r}20 \\
\hline \text { Total }\end{array}$

T A B LE IV

GROUP A: INDICATIONS FOR WEDGE RESECTION IN 20 PATIENTS (21 SIDES)

\begin{tabular}{|c|c|c|}
\hline Indication & No. & Recurrence \\
\hline 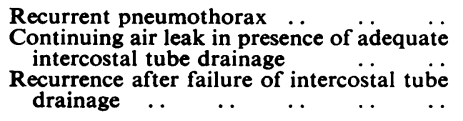 & $\begin{array}{r}15 \\
4 \\
2\end{array}$ & $\begin{array}{l}0 \\
0 \\
0\end{array}$ \\
\hline Total & 21 & 0 \\
\hline
\end{tabular}

There were no complications or deaths and no recurrences following wedge resection. One patient who later required a wedge resection on the $\vec{\theta}$ contralateral side is not included in this series as $P$ the follow-up period is only four years to date. In all but two patients treated for recurrence of pneumothorax, the first recurrence had occurred within one year of the primary episode.

GROUP B The lesions associated with the development of spontaneous pneumothorax are shown in Table V.

T A B L E V

GROUP B: ASSOCIATED LESIONS

\begin{tabular}{|c|c|c|c|c|}
\hline Lesion & & & & No. of Patients \\
\hline $\begin{array}{l}\text { Bronchitis and emphysema } \\
\text { Old healed tuberculosis } \\
\text { Asthma and bronchitis } \\
\text { Staphylococcal pneumonia .. }\end{array}$ & $\begin{array}{l}\cdots \\
\cdots \\
\cdots\end{array}$ & $\begin{array}{l}\cdots \\
\cdots \\
\cdots\end{array}$ & $\begin{array}{l}\cdots \\
\cdots \\
\cdots\end{array}$ & $\begin{array}{r}35 \\
13 \\
5 \\
1\end{array}$ \\
\hline Total & & & & 54 \\
\hline
\end{tabular}

There were 44 male and 10 female patients in the $\stackrel{\circ}{I}$ series.

Age range The range was from 29 to 84 years. Side affected The right side was affected in $44 \%$ and the left in 26 instances.

Management Initial intercostal tube drainage $\overbrace{\omega}$ was used. If tube management failed the patient 0 was treated by excision of bullae or pleurodesis (Tables VI and VII).

Late death There were seven deaths in the series. $\stackrel{\mathscr{D}}{\stackrel{+}{+}}$ Two patients died of cancer after six months and 7 one year, one died of uraemia after three years, $\frac{O}{\Phi}$ three died of chronic obstructive respiratory $\stackrel{\mathbb{D}}{\mathscr{D}}$ disease after four, five, and seven years, and one $\underset{\odot}{\mathbb{Q}}$ died in another city of contralateral pneumothorax after nine years. 
T A B L E V I

GROUP B: INITIAL TREATMENT OF 60 PNEUMOTHORACES

\begin{tabular}{|c|c|c|}
\hline Treatment & No. & Recurrence \\
\hline 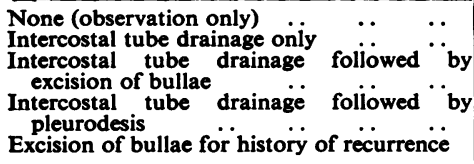 & $\begin{array}{r}1 \\
37 \\
6 \\
13 \\
3\end{array}$ & $\begin{array}{l}0 \\
6 \\
0 \\
2 \\
0\end{array}$ \\
\hline Total & 60 & 8 \\
\hline
\end{tabular}

There were no significant complications, no operative deaths, and no recurrences following excision of bullae. Of the two failures of pleurodesis one was later treated by excision of bullae and the other by resection of a tuberculous lobe.

T A B LE VII

GROUP B: OUTCOME OF 59 PNEUMOTHORACES TREATED BY INITIAL INTERCOSTAL TUBE DRAINAGE

\begin{tabular}{|c|c|c|}
\hline $\begin{array}{l}\text { Succeeded as only treatment } \\
\text { Continuing air leak required excision of bullae } \\
\text { pleurodesis } \\
\text { Late recurrence of pneumothorax } \ldots\end{array}$ & $\begin{array}{l}\ddot{\text { or }} \\
\cdots \\
\cdots\end{array}$ & $\begin{array}{r}31 \\
22 \\
6\end{array}$ \\
\hline Total & & 59 \\
\hline
\end{tabular}

Five recurrences occurred between three and six weeks after initial intercostal tube management and one occurred a year later. The recurrences were treated by excision of bullae in four patients, by intercostal tube drainage in one, and by resection of a tuberculous lobe in another.

\section{DISCUSSION}

Spontaneous pneumothorax may arise as a complication of a variety of lung disorders. The results of various forms of treatment, and the prognosis, will vary according to the widely differing lesions that may be associated with the pneumothorax. The aim of initial treatment is to relieve dyspnoea, to avoid the risk of tension pneumothorax, and to encourage early full lung expansion. Definitive treatment should be designed to take into account the underlying lung lesion and its propensity for producing recurrence of pneumothorax.

While bedrest alone has been advocated as a means of treatment when the pneumothorax is minimal, is not progressing, and is not associated with respiratory embarrassment, this approach takes no account of the inconvenience and economic loss to the patient or the burden on hospital resources that results from the prolonged period of treatment that is necessary.

Intercostal tube drainage is advocated for early management. In our series of group A patients, tube drainage revealed that the air leak was frequently sealed within hours of the onset of symptoms, and the patient was usually discharged from hospital within 48 hours. Tube drainage provides immediate safety, and in many patients no further treatment is required. Unrelieved, a continuing air leak may embarrass breathing or endanger life by the development of a tension pneumothorax. When respiratory reserve is already grossly impaired, as in advanced chronic bronchitis and emphysema, respiratory failure may occur very early after pneumothorax. Rapid and complete lung re-expansion is therefore mandatory if these patients are to be saved.

A plan is also required on which to base continuing treatment so as to reduce the duration and frequency of hospitalization and to abolish the sense of insecurity thereby engendered in the patient. Fortunately a good indication as to the prognosis can usually be obtained within 48 hours of the beginning of treatment. During this time the majority of patients will have completed their definitive treatment, and for the remainder a clear plan of management can be defined. Such a régime is possible because the course of the pneumothorax reflects the nature and potential for recurrence of the underlying lung disease. The severity of the basic pathological process declares itself; recurrence or persistent air leak indicate an extensive lesion such as multiple thinwalled cysts or large tears in cysts and the consequent need for more radical measures. This concept can be used as a guide to management so as to minimize failure of treatment.

In young subjects with no serious underlying lung disease apart from apical bullous cysts (group A patients) wedge resection was performed if adequate intercostal tube management had not resulted in closure of the lung leak within 48 hours, and in those patients with a history of previous pneumothoraces. This was deemed especially important where pneumothoraces had occurred on both sides, treatment being directed at making safe at least the side currently affected.

The findings at operation have been remarkably constant (Lichter and Gwynne, 1971). At the apex of the lung there is a small area of fibrosis surmounted by a cyst, or group of cysts, usually varying in size and measuring from $1-2 \mathrm{~mm}$ to $1 \mathrm{~cm}$ or more in diameter. A pin-hole opening at the summit of the cyst may sometimes be observed, but frequently such an opening is not visible and has presumably already sealed over. Occasionally the diseased area appears macroscopically as an area of fibrosis with an irregular 
crumpled pleural surface, not exhibiting obvious blebs. One or other of the lesions described has always been found at thoracotomy; the lesion measures $2-3 \mathrm{~cm} \times 1-2 \mathrm{~cm}$ and the remainder of the lung is entirely normal. The diseased area is recognized on inspection but its limits are determined by palpation, when the underlying fibrotic area can readily be detected in comparison with the subjacent normal lung. Histologically the changes in the diseased area are nonspecific but suggest postinflammatory fibrosis and cyst formation. The excised portion of lung shows focal and compensatory emphysema, atelectasis, fibrosis, chronic inflammation, pigment deposition in macrophages, alveolar cell proliferation, intrabronchial and peribronchial lesions, and vascular changes (endarteritis obliterans). On histological grounds alone the changes are best explained on the basis of postinflammatory disorganization, the inflammation being most likely due to nonspecific infection. The curious localization of the lesions, and the occurrence predominantly in young men, are obscure features which suggest some local inherent predisposition.

Pleurodesis and pleurectomy, which are designed to obliterate the whole pleural space, are condemned for group A patients. The discomfort and morbidity are usually greater than in patients undergoing thoracotomy and wedge resection, in whom convalescence is smooth and uneventful. The results of pleurodesis are uncertain, and there is at least a possibility that pulmonary function may be interfered with. In the event of the need for thoracotomy for some other condition at a later date, obliteration of the pleural space may well add to the hazards of the procedure. Wedge resection is preferred to pleurodesis and pleurectomy because it removes the whole of the diseased tissue, deals exclusively with the disease process, and leaves the normal lung untouched. It is thus uniformly successful and there is no need for pleurectomy or similar procedures to supplement excision of the diseased area.

In older patients with gross underlying lung disease, when the initial treatment is ineffective the problem is different from that in group $A$ patients; extensive disease, usually bilateral, does not permit of the same simple, safe, effective treatment that can be offered to the younger group of patients. For this reason tube drainage was persisted with for up to 14 days in the hope of avoiding operation. When tube drainage failed, thoracotomy and excision of bullae was the treatment of choice if the patient was fit for the procedure (deemed suitable only in nine of 60 patients as initial treatment and in a further four after recurrence). When thoracotomy was not feasible, chemical pleurodesis was employed.

Results have confirmed that the plan of man- $\frac{\bar{c}}{\bar{c}}$ agement outlined gives a high initial success rate $\overrightarrow{\mathbb{D}}_{\vec{D}}$ and reduces the frequency of readmission for recurrence of pneumothorax. Reported recur- $\omega$ rence rates in other series vary from $5 \%$ to $60 \% \overrightarrow{0}$ (Killen and Gobbel, 1968). The authors point out that the discrepancies in recurrence rate depend on $\vec{\sigma}$

(a) the length of the follow-up period;

(b) the fact that some series include suspected episodes and others only those radiologically $\tilde{C}_{0}$ demonstrated;

(c) the inclusion or otherwise of contralateral $\stackrel{\omega}{\sim}$ recurrences;

(d) whether or not the series includes previous episodes that occurred before the patient was first treated by the reporting observer; the? first episode observed by the investigator is sometimes designated the 'initial' episode $\overrightarrow{-}$ when such an episode may in fact have been a recurrence;

(e) the type of treatment used for the initial episode.

A further cause of confusion is that frequently no distinction is made between group $A$ and group B patients with their widely differing under- $\stackrel{\Phi}{\varnothing}$ lying pathologies.

In our series the value of intercostal tube $\frac{0}{3}$ drainage as initial management was demonstrated $\supset$ in group $\mathbf{A}$ patients as

(a) a safe measure (100\% success);

(b) a form of treatment which in itself was음 successful in $77 \%$ of patients;

(c) an indicator when more radical measures were needed-in $15 \%$ of patients treated by intercostal tube drainage who continued to $\cong$ leak air for more than $\mathbf{4 8}$ hours.

In group $B$ patients, intercostal tube drainage $\frac{\text { ? }}{2}$ alone was successful in $52.5 \%$ of patients.

Managed in the manner recommended, $95 \%$ 을 of group A patients and $87 \%$ of group B patients $N$ were either cured by tube drainage alone or treated definitively and lastingly during their first admis- $N$ sion to hospital. The chance of recurrence in $\underset{\omega}{N}$ patients so treated is therefore small enough to justify reliance upon this method. Exceptions may 0 be made in patients at special risk, such as airmen $\overparen{\Phi}$ and sailors.

Operation is performed only in selected patients; $\square$ the use of pleurodesis or pleurectomy is unwarranted in group A patients. The long-term $\stackrel{\Phi}{\overparen{D}}$ follow-up indicates that where success has been $\stackrel{\otimes}{\Phi}$ achieved within the first year of initial manage- $\bar{O}$ ment along the lines described, further recurrences are rare. 


\section{REFERENCES}

Baronofsky, I. D., Warden, H. G., Kaufman, J. L., Whatley, J., and Hanner, J. M. (1957). Bilateral therapy for unilateral spontaneous pneumothorax. Journal of Thoracic Surgery, 34, 310.

Carr, D. T., Silver, A. W., and Ellis, F. H., Jr. (1963). Management of spontaneous pneumothorax: with special reference to prognosis after various kinds of therapy. Proceedings of the Staff Meetings of the Mayo Clinic, 38, 103.

Gobbel, W. G., Jr., Rhea, W. G., Jr., Nelson, I. A., and Daniel, R. A., Jr. (1963). Spontaneous pneumothorax. Journal of Thoracic and Cardiovascular Surgery, 46, 331.

Hyde, L. (1962). Benign spontaneous pneumothorax. Annals of Internal Medicine, 56, 746.
Killen, D. A. and Gobbel, W. G. (1968). Spontaneous Pneumothorax. Churchill, London.

Lichter, I. and Gwynne, J. F. (1971). Spontaneous pneumothorax in young subjects. Thorax, 26, 409.

MacQuigg, R. E. (1955). Spontaneous pneumothorax: the case for early thoracotomy. American Surgeon, 21, 478.

Saha, D. (1964). Parietal pleurectomy for prevention of recurrent spontaneous pneumothorax. British Journal of Diseases of the Chest, 58, 78.

Sengupta, A. (1963). The treatment of recurrent spontaneous pneumothorax with iodine and talc poudrage. British Journal of Diseases of the Chest, 57, 197.

Wolcott, M. W., Shaver, W. A., and Jennings, W. D. (1963). Spontaneous pneumothorax: management by tube thoracostomy and suction. Diseases of the Chest, 43,78 . 